\title{
Applicability of condensation particle counters to measure atmospheric clusters
}

\author{
M. Sipilä ${ }^{1, *}$, K. Lehtipalo ${ }^{1}$, M. Kulmala ${ }^{1}$, T. Petäjä ${ }^{1}{ }^{* * *}$, H. Junninen ${ }^{1}$, P. P. Aalto ${ }^{1}$, H. E. Manninen ${ }^{1}$, E.-M. Kyrö ${ }^{1}$, \\ E. Asmi ${ }^{1}$, I. Riipinen ${ }^{1}$, J. Curtius ${ }^{2, * * *}$, A. Kürten ${ }^{3, * * *}$, S. Borrmann ${ }^{2,3}$, and C. D. O'Dowd ${ }^{4}$ \\ ${ }^{1}$ Department of Physics, University of Helsinki, Finland \\ ${ }^{2}$ Institute for Atmospheric Physics, J. Gutenberg-University Mainz, Mainz, Germany \\ ${ }^{3}$ Max Planck Institute for Chemistry, Particle Chemistry Department, Mainz, Germany \\ ${ }^{4}$ School of Physics \& Centre for Climate and Air Pollution Studies, Environmental Change Institute, National University of \\ Ireland, Galway, Ireland \\ *also at: Helsinki Institute of Physics, Helsinki, Finland \\ ** now at: Earth and Sun Systems Laboratory, Atmospheric Chemistry Division, National Center for Atmospheric Research, \\ Boulder, USA \\ *** now at: Institute for Atmosphere and Environment, J. W. Goethe-University Frankfurt, Frankfurt am Main, Germany \\ ${ }^{* * * *}$ now at: Division of Engineering and Applied Science, California Institute of Technology, Pasadena, CA, USA
}

Received: 19 November 2007 - Published in Atmos. Chem. Phys. Discuss.: 3 March 2008

Revised: 26 May 2008 - Accepted: 10 June 2008 - Published: 25 July 2008

\begin{abstract}
This study presents an evaluation of a pulse height condensation particle counter (PH-CPC) and an expansion condensation particle counter (E-CPC) in terms of measuring ambient and laboratory-generated molecular and ion clusters. Ambient molecular cluster concentrations were measured with both instruments as they were deployed in conjunction with an ion spectrometer and other aerosol instruments in Hyytiälä, Finland at the SMEAR II station between 1 March and 30 June 2007. The observed cluster concentrations varied and ranged from some thousands to $100000 \mathrm{~cm}^{-3}$. Both instruments showed similar (within a factor of $\sim 5$ ) concentrations. An average size of the detected clusters was approximately $1.8 \mathrm{~nm}$. As the atmospheric measurement of sub 2-nm particles and molecular clusters is a challenging task, we conclude that most likely we were unable to detect the smallest clusters. Nevertheless, the reported concentrations are the best estimates to date for minimum cluster concentrations in a boreal forest environment.
\end{abstract}

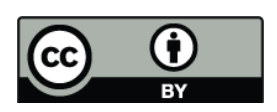

Correspondence to: M. Sipilä

(mikko.sipila@helsinki.fi)

\section{Introduction}

An important phenomenon associated with an atmospheric aerosol system is a formation of new atmospheric aerosol particles (Kulmala, 2003). Once formed, the aerosol particles need to grow further to sizes larger than $50-100 \mathrm{~nm}$ in diameter after which they are able to influence climate, even though smaller particles may have influences on human health and atmospheric chemistry. The atmospheric aerosol formation involves a complicated set of processes that include production of nanometer-size clusters from gaseous vapours, growth of these clusters to detectable sizes, and their simultaneous removal by coagulation with pre-existing aerosol particle population (e.g. Kerminen et al., 2001; Kulmala, 2003). While the aerosol formation has been observed to take place almost everywhere in the atmosphere (Kulmala et al., 2004a), serious gaps in our knowledge regarding to this phenomenon still exist. These gaps include existence and dynamics of atmospheric molecular clusters, vapours participating on atmospheric cluster formation, the effect of those clusters on atmospheric nucleation, the effect of ions on particle formation and also various impacts of the new particle formation on atmospheric chemistry, climate, human health and environment.

Published by Copernicus Publications on behalf of the European Geosciences Union. 
Although some field studies in a boreal forest environment have been promising (see e.g. Kulmala et al., 2005a), critical clusters formed during the atmospheric nucleation events have not been measured quantitatively until recently (Kulmala et al., 2007a) due to instrumental limitations. Only some measurements of the clusters during the nucleation events have been reported. Weber et al. (1995) showed that the clusters were present when $2.7-4 \mathrm{~nm}$ particles were detected. Kulmala et al. (2005a) proved the existence of clusters during a nucleation event but even more interestingly they showed that the clusters exist practically all the time. Recently, Kulmala et al. (2007a) observed neutral clusters and the initial state of atmospheric nucleation. However, due to the fact that measurements in sub- $2 \mathrm{~nm}$ size range are challenging, more measurements, and preferably using different instruments, are needed to verify those observations.

There are several points that make detection of sub- $3 \mathrm{~nm}$ particles complicated. First, since the small clusters are extremely diffusive, transport efficiency from the atmosphere to the instrument decreases rapidly with a decreasing particle size. Second, interaction of small neutral clusters with their surroundings is very weak. They do not obtain charge very efficiently. Thus, electrical detection of the charged clusters of low concentration is problematic because of a poor signal to noise ratio. Condensation based techniques allow one to activate and detect neutral clusters below $2 \mathrm{~nm}$ in diameter (Winkler et al., 2008). However, there are several technical difficulties in the application of these techniques to the field measurements. In this paper we show how those challenges can be overcome.

Atmospheric aerosol formation is strongly coupled with chemistry, particularly with the formation of sulphuric acid and other vapours of very low volatility such as multifunctional organic compounds and iodine vapours (Curtius, 2006). Pre-existing aerosol particles act as a sink for these vapours and nucleated clusters, thus inhibiting the atmospheric aerosol formation. The aerosol formation seems to be related also to several meteorological parameters and phenomena, including solar radiation and atmospheric mixing processes such as the evolution of the continental boundary layer or the mixing of stratospheric and tropospheric air near the tropopause (Lyubovtseva et al., 2005).

In this paper we present the applicability of two condensation particle counter (CPC) systems to atmospheric molecular cluster measurements. We use these CPC systems in their improved mode to observe quantitatively both the concentration and the size of atmospheric neutral clusters. The first CPC system is a modified TSI 3025A ultra-fine condensation particle counter with white light optics (Marti et al., 1996; Dick et al., 2001) and the second one is an adiabatic expansion type CPC (Kürten et al., 2005). We conducted a set of laboratory tests with the CPC systems to assure their functionality to detect cluster sized objects and we also present atmospheric cluster concentrations measured at a boreal forest site.

\section{Condensation particle counter cystems}

\subsection{General approach}

The two CPC systems used in this study were characterized with laboratory generated nanoparticles. A tungsten oxide generator (Grimm 7860, Grimm Aerosol Technik, GmbH) was used as a particle source at sizes down to mobility diameters of approximately $1.5 \mathrm{~nm}$. Generated particles were charged with a radioactive Am-241 neutralizer (activity 60 $\mathrm{MBq}$ ). A similar neutralizer was also used to generate ion clusters and neutral sub- $2 \mathrm{~nm}$ recombination clusters. A short (109 mm) Vienna-type Differential Mobility Analyzer (DMA, Winklmayr et al. 1991) was used to classify the generated particles with a sheath flow of $20 \mathrm{lpm}$ and an aerosol flow from 1.5 to $2.5 \mathrm{lpm}$ while a positive high voltage was applied to the DMA. A TSI-3068 electrometer was used as a reference for negatively charged clusters.

The CPC systems were also tested at atmospheric conditions. Field measurements were carried out during a EUCAARI (European Integrated project on Aerosol Cloud Climate and Air Quality Interactions) 2007 measurement campaign at the Hyytiälä forestry field station (SMEAR II, Hari and Kulmala, 2005), Southern Finland, from March to June 2007. This study focuses on the measurements by a pulse height CPC and an expansion CPC, but utilizes also supporting data from a Differential Mobility Particle Sizer DMPS (Aalto et al., 2001) and from a Balanced Scanning Mobility Analyzer, BSMA (Tammet 2004; 2006). The DMPS measures particle size distributions from $3 \mathrm{~nm}$ up to $1 \mu \mathrm{m}$. The BSMA measures ion mobility spectra in the mobility range of $3.2-0.032 \mathrm{~cm}^{2} \mathrm{~V}^{-1} \mathrm{~s}^{-1}$ which corresponds to mobility equivalent diameter range of ca $0.8-8 \mathrm{~nm}$.

\subsection{Pulse height condensation particle counter (PH-CPC)}

The first instrument, which applicability was investigated was the pulse height CPC. It exploits an axial gradient of butanol supersaturation inside a condenser of the CPC. Particles entering the condenser activate for growth at the different axial positions depending on their size resulting in a monotonic link between the initial particle size and the final droplet size for the initial particle sizes smaller than ca $15 \mathrm{~nm}$ (Saros et al., 1996). Given that the scattering cross-section for a white light is a monotonic function of a droplet size, measuring the intensity of the scattered light with a multi-channel analyzer (MCA) gives information about the initial particle size. The pulse height analysis method has been used in size distribution measurements between 3 and $10 \mathrm{~nm}$ (Weber et al., $1995 ; 1998)$ as well as to determine composition of freshly nucleated nanoparticles (O'Dowd et al., 2002; Hanson et al., 2002).

A detection efficiency as a function of particle size in a conductive cooling type condensation particle counter is determined mainly by the particle transport efficiency from the 
inlet to the activation region inside the condenser, and by the saturation ratio of condensing vapour. The detection efficiency of the commercial CPCs can be improved to some extent by increasing the supersaturation inside the condenser (see e.g. Mertes et al., 1995; Petäjä et al., 2006). Kulmala et al. (2007b) improved the 50\% cut-off size of UF-02proto CPC from nominal $4.5 \mathrm{~nm}$ down to $2 \mathrm{~nm}$ with this technique. However, increasing the supersaturation too much induces homogeneous nucleation of the working fluid inside the condenser. This leads to excess counts in the optical detector due to the homogeneously nucleated particles. This problem can be partly solved by applying a pulse height $(\mathrm{PH})$ analysis of white light scattered by the droplets grown in the condenser (Saros et al., 1996).

We utilized the PH technique to distinguish between the homogeneously formed butanol droplets and the droplets formed by the heterogeneous nucleation on the molecular cluster sized objects inside the condenser. The PH-CPC used in this study is a TSI-3025A conductive cooling type CPC (Stolzenburg and McMurry, 1991) with modified optics (Dick et al., 2000) and a multi-channel analyzer. To maximize the activation probability of the smallest clusters, butanol supersaturation was increased by elevating the saturator temperature from normal $37^{\circ} \mathrm{C}$ up to $43-44^{\circ} \mathrm{C}$ and decreasing the condenser temperature by $0-2^{\circ} \mathrm{C}$ from the nominal $10^{\circ} \mathrm{C}$ set point.

A concentration of the particles entering the $\mathrm{PH}-\mathrm{CPC}$ has an effect on the detection efficiency. According to Saros et al. (1996) a primary reason for this is a dead time in the MCA. As more particles enter the system, there is not enough time for the MCA to recover from the previous signal. This leads to the dead-time, when neither pulse height nor concentration can be measured. A secondary reason for the lowered detection efficiency of the PH-CPC in the high concentrations is butanol vapour depletion. In the high concentrations there are more particles competing for the same amount of vapour in the condenser. As a result, the particles reach smaller sizes and some of the smallest particles might not even activate. Thus, in larger than $4000 \mathrm{~cm}^{-3}$ aerosol number concentrations Saros et al. (1996) suggested a dilution system to be placed in front of the PH-CPC. This ensures that the MCA will have enough time to recover, the butanol is not depleted significantly and coincidence is minimal. To maximize the cluster concentration inside the PH$\mathrm{CPC}$ condenser we conducted all measurements without dilution, as the typical background aerosol number concentrations in Hyytiälä are below $4000 \mathrm{~cm}^{-3}$. The concentrations during nucleation events and pollution episodes, however, usually exceed $4000 \mathrm{~cm}^{-3}$ and therefore the data associated with these events of elevated concentrations are not very reliable.

Because the condensation sink caused by the activated particles affects, besides the detection efficiency, also homogeneous nucleation rate, we applied a diffusion battery (modified from TSI-3042) in front of the PH-CPC. This allowed us to determine a fraction of homogeneous nucleation in the presence of larger, few nanometers and up, particles in the observed pulse height spectrum. We used only four stages of the diffusion battery; and the fourth stage was assumed to remove practically all cluster sized objects and the resulting distribution in the lower MCA channels was assumed to be solely due to the homogeneous nucleation. An additional fifth stage, an absolute filter (HEPA, Pall Corporation) was used to remove all particles as well as molecular clusters, and in this case all detected pulses were due to the homogenous nucleation. Because of reasonably high losses already in the first stage of the diffusion battery, measurements with a free inlet were also conducted.

A particle penetration through the diffusion battery to the $\mathrm{PH}-\mathrm{CPC}$ was characterized in the laboratory with a tungsten oxide generator and a DMA setup described above. An average size $d_{p}$ of the clusters (activated in the condenser) can be determined from

$$
\begin{aligned}
& \Omega_{(i-j) /(k-l)}\left(d_{p}\right)= \\
& \frac{p_{i}\left(d_{p}\right)-p_{j}\left(d_{p}\right)}{p_{k}\left(d_{p}\right)-p_{l}\left(d_{p}\right)}=\frac{N_{\mathrm{hom}}+N_{i}-\left(N_{\mathrm{hom}}+N_{j}\right)}{N_{\mathrm{hom}}+N_{k}-\left(N_{\mathrm{hom}}+N_{l}\right)},
\end{aligned}
$$

where $\Omega_{(i-j) /(k-l,)}\left(d_{p}\right)$ is an experimentally determined function which describes the relations of the different diffusion battery stages, $p_{i, j, k, l}\left(d_{p}\right) \mathrm{s}$ are the penetrating fractions through the diffusion battery stages $i, j, k$ and $l$ as a function of particle diameter $d_{p}, N_{i, j, k, l}$ are the detected concentrations after the corresponding stages with the signal from the large particle activation subtracted, and $N_{\text {hom }}$ is the signal due to the homogeneous nucleation which is assumed to be constant during one diffusion battery cycle.

Charged ion clusters are present in variable concentrations in the atmosphere (e.g. Hirsikko et al., 2005; Kulmala and Tammet, 2007). In order to observe a signal due to the ion clusters, we applied an ion filter to the inlet of the PH-CPC. The ion filter was a $4 \mathrm{~cm}$ coaxial tube (inner diameter. $8 \mathrm{~mm}$ ) with a $32 \mathrm{~V}$ voltage between the electrodes. Electric field was switched on and off during the measurements. The difference between the two PH-spectra revealed the concentration of the ion clusters.

\subsection{Expansion CPC}

The second instrument to evaluate for applicability to atmospheric cluster measurements was the expansion CPC. In this instrument a rapid adiabatic expansion of gas-particle mixture leads to super-saturated conditions and subsequently activation of the particles as the super-saturated vapour condenses onto the sampled particles. A more detailed discussion of the expansion technique can be found in Wagner (1985). The expansion CPC (E-CPC) used in this study was a modified version of the one described by Kürten et al. (2005). As the condensing vapour we used both water and butanol. Sample flow rate of the E-CPC was 2 litres 


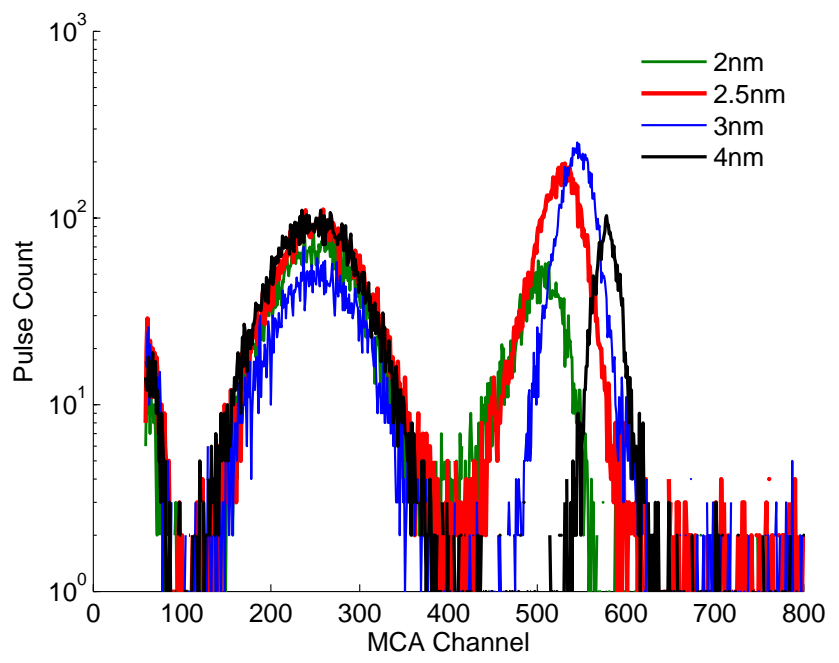

Fig. 1. Pulse height spectra for different sizes of $\mathrm{WO}_{\mathrm{x}}$ particles. Homogenous nucleation appears as a Gaussian shaped $\mathrm{PH}$-spectrum between the channels $\sim 100$ and 400 . Activated $\mathrm{WO}_{x}$ particles are seen in the channels 400-600. Initially larger particles yield pulses in the higher channels. Pulse count is a total number of the pulses counted during an integration time (here $45 \mathrm{~s}$ ), and can be taken as an arbitrary unit.

per minute $(\mathrm{lpm})$. A smaller flow $(0.8 \mathrm{lpm})$ of particle free air saturated with the condensing vapour is mixed with the sample flow. This mixture is then directed to an expansion chamber. After an adiabatic expansion, particles within the sample are activated and grow to sizes where they scatter visible light from a laser beam. In this instrument the amount of light forward-scattered $\left(1.1-4.4^{\circ}\right)$ by the droplets inside the expansion chamber is measured as a function of time. Growing droplets scatter light depending on their size according to the Mie-theory and the concentration of droplets can be calculated from the scattering intensity maxima. We used a second Mie maximum for the calculation of the total number concentration of the activated particles.

By scanning the expansion ratio (E) and thus changing the supersaturation inside the expansion chamber we were able to activate particles of different sizes. The expansion scans were performed with a free inlet and with a set of diffusion tubes. We used three parallel $4 \mathrm{~mm}$ inner diameter $1 \mathrm{~m}$ long copper tubes with a flow rate of ca. $0.7 \mathrm{lpm}$ through each of the inlets. The tubes had approximately a 50\% penetration efficiency for $3.5 \mathrm{~nm}$ particles according to GormleyKennedy diffusion loss calculations (e.g. Baron and Willeke, 2001). Tube surfaces were assumed to be in equilibrium with the ambient water vapour concentration. These tubes were used to screen out the smallest clusters. In some measurements an ion filter similar to one used together with the $\mathrm{PH}-\mathrm{CPC}$ was applied to detect the signal caused by the ion clusters.
To test the applicability of the E-CPC for atmospheric cluster measurements we generated ions and neutral recombination clusters with an Am-241 charger in conjuction with an ion filter. These experiments were conducted using water as the condensing vapour in the E-CPC.

\section{Results}

\subsection{Laboratory experiments}

\subsubsection{Pulse height CPC in homogenous nucleation regime}

As we increased a temperature difference between the saturator and the condenser of the $\mathrm{PH}-\mathrm{CPC}$, homogenous nucleation occurred inside the instrument. With negatively charged tungsten oxide particles we tested a limit where the droplets formed via heterogeneous nucleation on the sampled particles could no longer be discriminated from the homogeneously nucleated butanol droplets. The resulting pulse-height spectra are presented in Fig. 1. The homogenously nucleated droplets were clearly distinguishable from the droplets nucleated heterogeneously on the negatively charged $\mathrm{WO}_{\mathrm{x}}$ calibration particles at least down to $2 \mathrm{~nm}$. It should be emphasized that since, e.g., butanol solubility of the artificial $\mathrm{WO}_{\mathrm{x}}$-particles is certainly different from the atmospheric particles, these laboratory spectra cannot be directly compared to the atmospheric data.

Attached charge lowers a supersaturation needed for activation (Winkler et al., 2008). Therefore the results for negatively charged clusters are not necessarily representative of electrically neutral clusters. Unfortunately a generation of neutral sub-3 nm clusters of a known composition, size and concentration is a very challenging task due to non-existent reference instrument and therefore a complete laboratory verification of the effect of charge is left out of the scope of this study. However, to get an indication where the pulses from the ambient neutral sub- $2 \mathrm{~nm}$ clusters would appear, we generated neutral clusters by recombination of ions produced by radioactive decay. Inside an Am-241 source a vast number of ions are generated. Some of these ions recombine and form electrically neutral stable clusters.

Without electric filtration of the sample air a fraction of ions and neutral clusters formed in collisions between positive and negative ions were activated inside the PH-CPC. Total number concentration of the activated clusters in this experiment was ca. $6000 \mathrm{~cm}^{-3}$. When the ions were filtered away using a co-axial electric filter described above, the neutral clusters were clearly visible and distinguishable from homogeneous nucleation, even though their pulse height $(\mathrm{PH})$ distribution partly overlaps with the $\mathrm{PH}$-distribution of the homogenously nucleated droplets (Fig. 2). The PHdistribution of the homogeneously nucleated droplets was determined by applying a diffusion tube to remove molecular clusters from the sample flow. This measurement was 


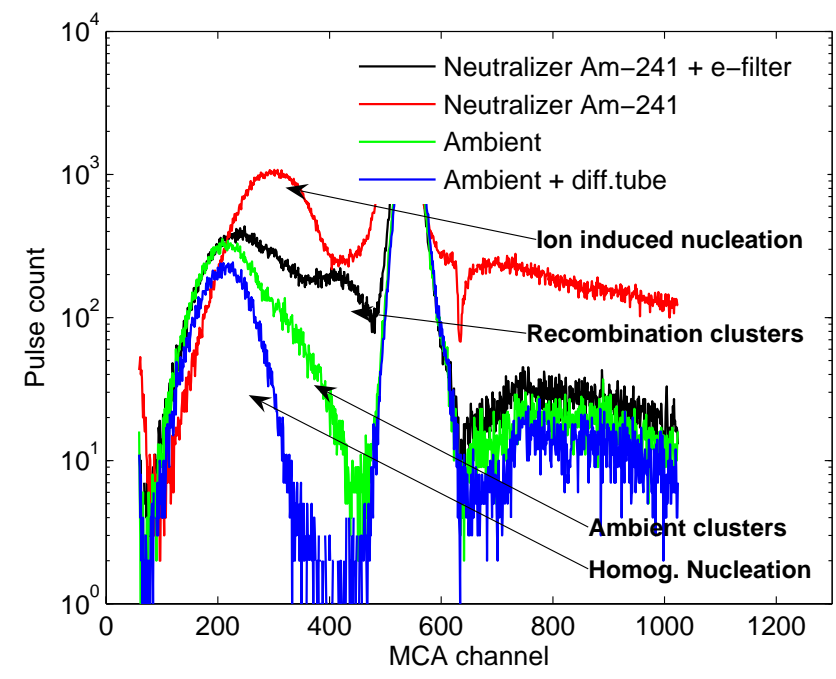

Fig. 2. Pulse height spectra in indoor air (green line) and in indoor air pre-treated by removing clusters with an aid of a diffusion tube (blue) as well as pulse height spectra of bipolar ions generated in an $\mathrm{Am}^{241}$-source (red) and neutral recombination clusters (black) are depicted.

carried out with urban indoor air also containing aerosol particles, which were accumulating in the MCA channels 500700. Also as a first indication of atmospheric neutral clusters, a shoulder was clearly seen in the Gaussian-shaped homogeneous nucleation spectrum in the ambient unhandled sample (green line in Fig. 2).

\subsubsection{PH-CPC with a Diffusion Battery}

An example of the diffusion battery function is shown in Fig. 3. $\Omega_{(0-1) /(1-2)}$ approaches unity with particle diameters larger than $3 \mathrm{~nm}$. This shows that practically all particles larger than $3 \mathrm{~nm}$ penetrate through diffusion battery stages 0 2 . With the particle sizes below $2 \mathrm{~nm} \Omega_{(0-1) /(1-2)}$ increases rapidly showing that more and more particles are removed already in the stage 1 . By determining $\Omega_{(0-1) /(1-2)}$ from field data a corresponding cluster diameter can be obtained (see Sect. 3.2.).

\subsubsection{Expansion CPC}

In laboratory tests with the expansion CPC, purified water (Milli-Q) was used as the condensing vapour. Larger the expansion ratio, smaller particles activate until homogeneous nucleation of water vapour occurs. Kürten et al. (2005) have shown that a $50 \%$ cut-off size below $3.5 \mathrm{~nm}$ can be achieved with the expansion CPC. Here we will show that even smaller particles can be detected with a reasonably high efficiency.

To test the E-CPC for a polydisperse ambient aerosol population we varied the expansion ratio (E) from 1.4 to 2.5. Activation of a background aerosol population occurred around $\mathrm{E}=1.5$ and an onset of homogeneous nucleation was seen

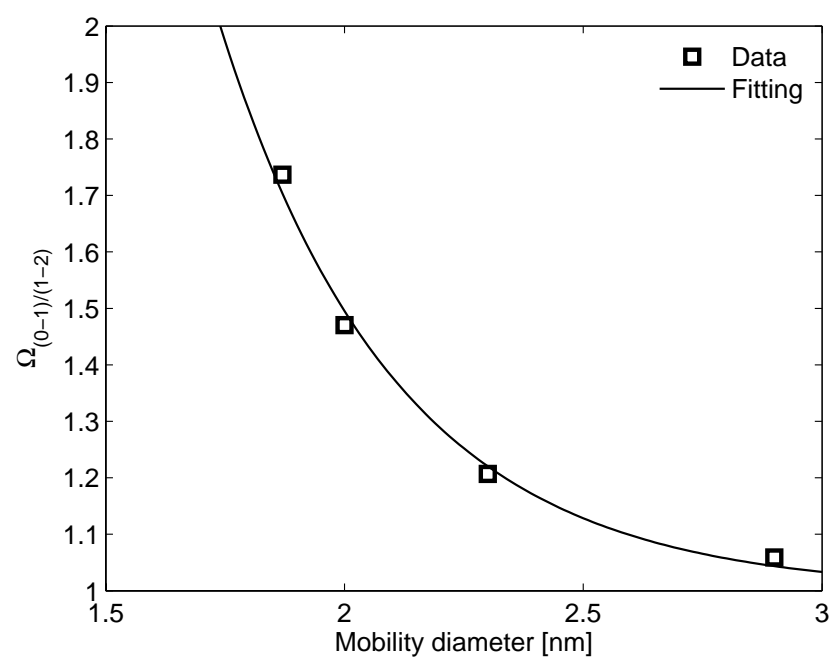

Fig. 3. Experimentally determined diffusion battery function (see Eq. 1) describing the relations of stages 0,1 and $2 . \Omega_{(0-1) /(1-2)}$ can be obtained from the field data and the average diameter solved using this function.

around $\mathrm{E}=2.3$ (Fig. 4). Ions, however, as well as neutral clusters will activate well before that. We tested the onset expansion ratios for Am-241 generated ion clusters by placing the radioactive neutralizer in front of the E-CPC. With this setup the onset took place at $\mathrm{E}=1.9$. After filtering the ions the onset moved approximately up to $\mathrm{E}=2.0$, which is close to the value where the onset for homogenous nucleation was observed with a diffusion tube.

Cluster concentration can be calculated as a differences in measured concentrations with and without a diffusion tube and an ion filter. The concentration of all the activated clusters (charged and neutral) is the difference between the data obtained with the charger attached to the E-CPC and the concentration detected with the diffusion tube (base signal). A concentration of ca. $4 \cdot 10^{4} \mathrm{~cm}^{-3}$ was detected (Fig. 4). The concentration of the neutral clusters, on the other hand, is the difference between the readings with the charger - ion filter combination and the diffusion tube (ca. $2 \cdot 10^{4} \mathrm{~cm}^{-3}$ ). The concentration of the ion clusters in this experiment is thus the difference between the total cluster concentration and the neutral cluster concentration and it is in the order of $2 \cdot 10^{4} \mathrm{~cm}^{-3}$. A comparison of the detected total cluster concentration $\left(\sim 4 \cdot 10^{4} \mathrm{~cm}^{-3}\right)$ to a corresponding signal detected by PH-CPC $\left(\sim 6000 \mathrm{~cm}^{-3}\right)$ show that the detection efficiency of the E-CPC is approximately 6-7 times higher than the detection efficiency of the PH-CPC for these clusters.

A reason why measured concentrations fall off after a maximum in Fig. 4 is that activated clusters significantly consume condensing vapour when present in high concentrations. Thus, homogeneous nucleation is prevented and the concentration obtained by a simple subtraction is not valid. 

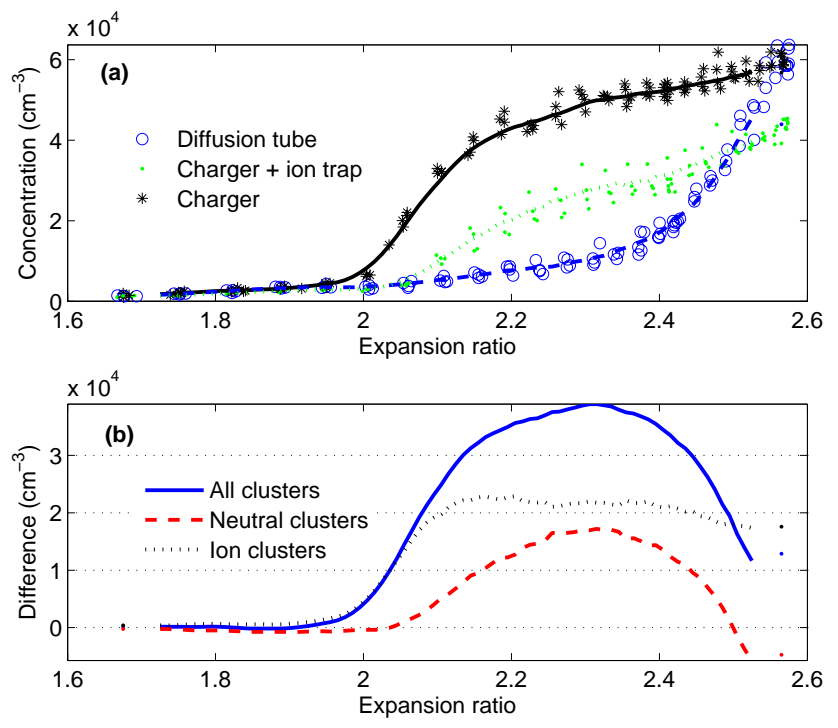

Fig. 4. Expansion scan experiment using bipolar ions generated with an $\mathrm{Am}^{241}$-source, and neutral recombination clusters (a). Measurement was carried out with ambient indoor air using water as a condensing vapour. A diffusion tube was used to remove natural clusters from the sample. Subtraction of the signals yields concentrations of neutral and ion clusters (b).

\subsection{Ambient cluster concentrations}

In order to evaluate their capability to detect atmospheric clusters both the Pulse-Height CPC and the expansion CPC were deployed on a field campaign in Hyytiälä, Finland. Measurements were conducted between 1 March and 30 June 2007. PH-CPC, BSMA and DMPS instruments were running throughout the whole campaign. E-CPC measurements were mainly carried out during May-June, 2007. Out of those campaign days, three days were selected for a closer examination. One of the days was a new particle formation event day (4 April 2007) and on two days (28 May, and 12 June 2007) no newly formed particles were observed.

Data interpretation relating a specific MCA channel directly to a particle size is a complicated task. Channels in the pulse height spectra are drifting all the time due to e.g. changes in intensity of the white light source, total particle concentration, and small fluctuations in flows and temperatures. Because the pulse height also depends on the particle composition (O'Dowd et al., 2002), coupling the channels to the particle sizes becomes even more difficult. Yet another difficult task is a determination of the absolute concentration of sub- $3 \mathrm{~nm}$ particles from the data, since the supersaturation and thus the activation probability strongly depends on the continuously changing ambient total particle concentration. Therefore these results are still to some extent qualitative. However, order of magnitude estimates can be given.

Calibration of the instrument for neutral sub-2nm particles and clusters is also a very challenging task as there is no reference instrument nor is the composition of the ambient clusters known. Therefore, to get an estimation of the neutral cluster detection efficiency we investigated the detection efficiency of the PH-CPC for atmospheric ion clusters. The detection efficiency was obtained by switching the ion filter electric field on and off and comparing the ion cluster concentration detected by the PH-CPC to the ion cluster concentration measured by the BSMA. This gives an upper boundary estimation for the detection efficiency of the neutral clusters under the assumption that the neutral cluster activation probability does not exceed the ion activation probability. However, the ion cluster detection efficiency fluctuated considerably due to the changes in the supersaturation inside the condenser. The highest measured detection efficiencies for the ion clusters were approximately $3 \%$ whereas the lowest records were buried in the noise, being practically $0 \%$. A typical size of negatively charged cluster ions according to the BSMA was $0.7-1.4 \mathrm{~nm}$, positive ones being slightly larger from 1 up to ca. $1.6 \mathrm{~nm}$ (in mobility equivalent diameter). To get an accurate estimation for the neutral cluster detection efficiency the efficiency for ions should have been recorded continuously. Therefore the most reliable data are associated with the ion detection efficiency measurements, and the three example days are presented below.

\subsubsection{A particle formation event day}

On 4 April 2007 new particle formation occurred in Hyytiälä. A few hours after the sunrise a new mode of atmospheric particles was detected with the Differential Mobility Particle Sizer (DMPS), which has a lower detection limit of $3 \mathrm{~nm}$. Meanwhile the total number concentration increased by an order of magnitude from $10^{3}$ to $10^{4} \mathrm{~cm}^{-3}$ (Fig. 5).

Taking a closer look on the PH-spectra revealed that the first steps of nucleation are recorded with the PH-CPC. Fig. 6 shows the development of the $\mathrm{PH}$-spectrum during an event day as a series of snapshots taken at 06:00, 08:00, 10:30 and 11:00 local time. Throughout the day there was a clear bimodal distribution in the lower MCA channels and a dominating peak at higher channels. The lower MCA channels around 200-300 represent homogeneously formed butanol droplets and the largest peak at around 700-800 is the signal from ambient aerosol particles larger than $\sim 10 \mathrm{~nm}$ in size. The droplet concentration due to the homogeneous nucleation was measured using the diffusion battery from time to time (two to four times a day) and outside these times we relied on the symmetry of the pulse height spectrum of the pure homogeneous butanol nucleation. Subtracting the contribution of the homogeneous nucleation leaves a residual mode in the channels from 300 to 500 . The pulse count in this residual mode before and after the nucleation event corresponds to ambient concentration of $40-100 \mathrm{~cm}^{-3}$ if the detection efficiency is not accounted for. If the detection efficiency of $2 \%$ is assumed (see next chapter for discussion 


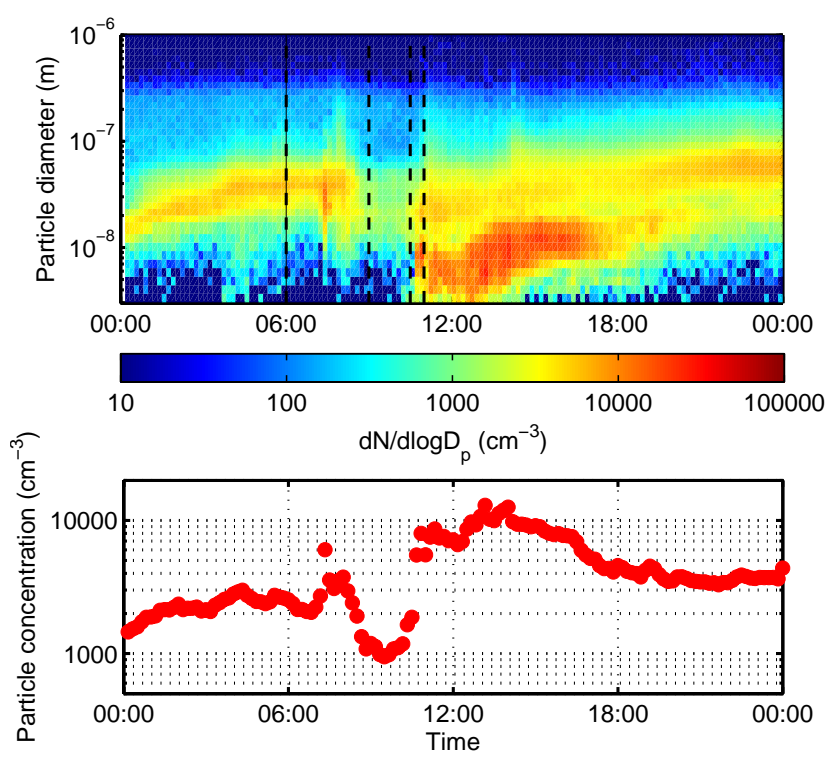

Fig. 5. Nucleation event recorded with a DMPS on April 4, 2007. Vertical lines indicate the times when the spectra in Fig. 6 (a-d) are measured. Boundary layer mixing after the sun rise dilutes the particles and induces a concentration minimum around 08:00-10:00.

on the detection efficiency), ambient concentration of 2000 $5000 \mathrm{~cm}^{-3}$ is needed to explain the observed signal.

At 09:00 (Fig. 6b) over an hour before new particle formation is detected with the DMPS (ca. 10:15), the particles start to appear between the MCA channels 400-600. When the event starts to be visible in the DMPS, homogeneous nucleation signal disappears rapidly from the $\mathrm{PH}$-spectrum due to the increasing particle concentration and subsequent depletion of butanol vapour. Hence, also the activation probability of the smallest clusters decreases and the detection efficiency drops.

Both the laboratory and the field measurements with the diffusion battery showed that homomolecular homogeneous nucleation always appears as a Gaussian-shaped pulse height distribution in PH-spectra (Fig. 1). Ambient data, however, typically showed a clear bimodal distribution (Fig. 6a) between the MCA channels 100-500 throughout a several month measurement period even though (and especially when), according to the DMPS, no particles between $3 \mathrm{~nm}$ and $10 \mathrm{~nm}$ were present. Also, the laboratory tests showed that co-incidence would not occur with that level of homogeneous nucleation. This fact proves that besides homomolecular butanol self-nucleation and large particle activation, a third process takes place in the supersaturated butanol aerosol mixture.
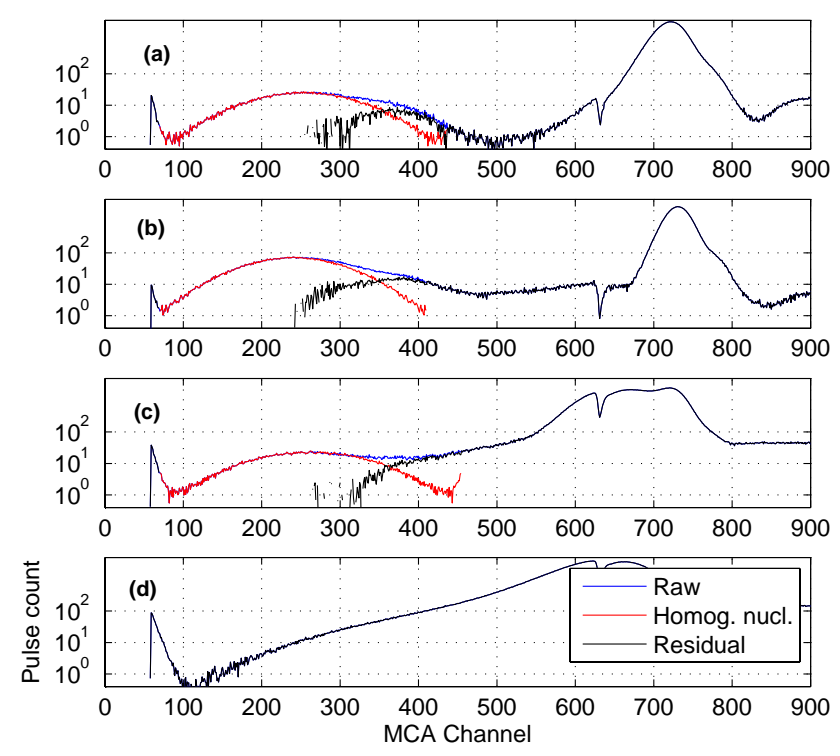

Fig. 6. Pulse height spectra recorded during an event day (4 April 2007) at 06:00 (a), 09:00 (b), 10:30 (c) and 11:00 (d). Blue line shows raw-data, red line represents a $\mathrm{PH}$-spectrum from homogeneous nucleation and black line describes the PH-spectrum after a subtraction of the homogeneous nucleation signal. A cluster mode is seen in channels between 300 and 500 (6a). Large particles are accumulating in the channels 600-800. Approximately an hour before the beginning of the nucleation event is detected with the DMPS (see Fig. 5), freshly nucleated particles start to appear between the channels 400 and 600 (6b). The increasing number concentration decreases the supersaturation most probably affecting the detection efficiency $(\mathrm{c}-\mathrm{d})$.

\subsubsection{Non event days}

\section{May 2007}

Figure 7 shows one experiment from 28 May, 2007 where measurements were conducted with the free inlet (blue curve), with the ion filter (black curve), and with the diffusion tube removing all of the clusters (red curve, 50\%cut-off at $3.5 \mathrm{~nm}$ ). The ion cluster detection efficiency is ca. $3 \%$ in this case based on a comparison with the BSMA $\left(1100 \mathrm{~cm}^{-3}\right)$. Utilizing this, a total cluster concentration here would be at least $5200 \mathrm{~cm}^{-3}$. Notably, the level of homogeneous nucleation (red curve, this measurement is conducted in lower supersaturation than the experiment presented in Fig. 6) is reasonably low compared to the signal from the activating clusters, indicating that increasing the supersaturation after the onset of homogeneous nucleation in presence of background aerosol does not anymore amend the detection efficiency significantly, but the nanoparticle transport losses from the inlet to the condenser start to dominate.

Besides large fluctuations in the detected signal, also an average size, determined from the diffusion battery measurements using equation 1 showed a quite unstable behaviour. 

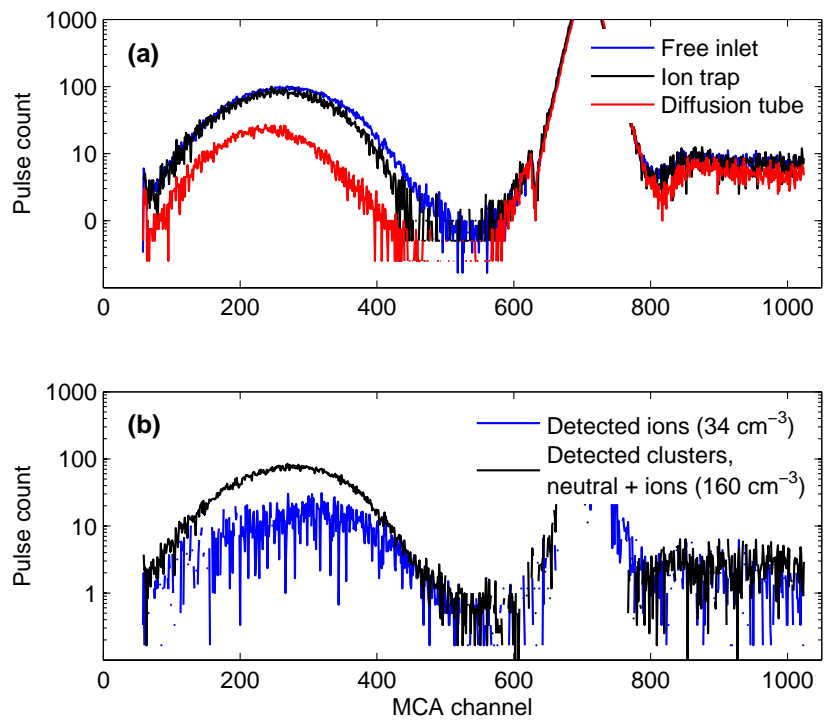

Fig. 7. (a) Example of pulse height distribution measured on 28 May 2007 with a free inlet (blue), with an ion filter (black) and with a diffusion tube (red) in the inlet. (b) Resulting distributions after subtracting the ion filter data (blue) and the diffusion tube data (black) from the free inlet data.
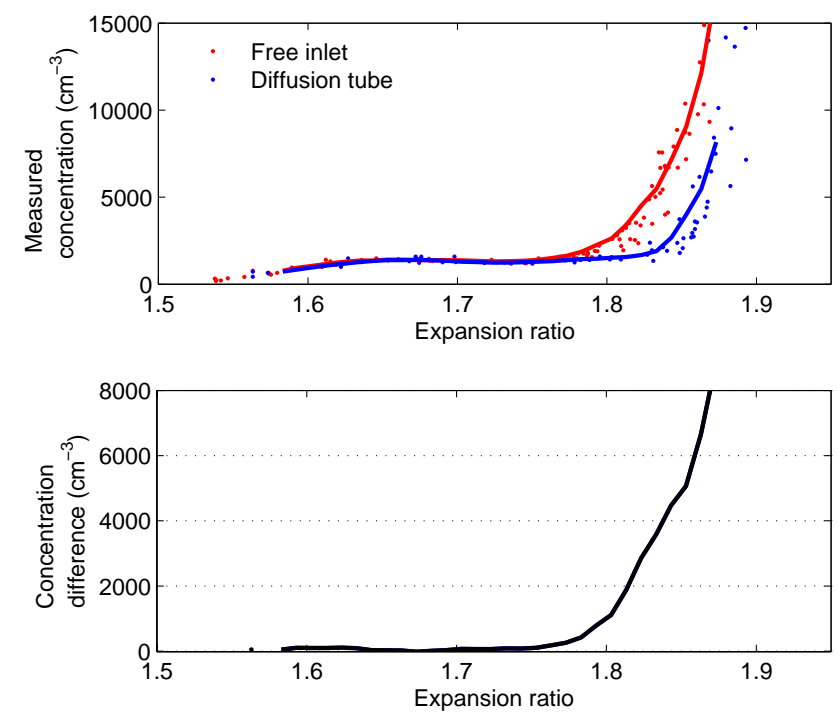

Fig. 8. Expansion scan experiment on 28 May 2007 at 07:50-08:20. Butanol is used as a condensing vapour. The upper panel shows detected signal with a free inlet (red dots) and with diffusion tubes (blue dots). Data resulting from a subtraction of the two signals is presented in lower panel.

An average signal ratio $\Omega_{(0-1) /(1-2)}$ for June was 1.774 with a standard deviation of 0.789 which corresponds to an average cluster mobility diameter of approximately $1.8 \mathrm{~nm}$ (see Fig. 3). As a comparison, measurement with artificial charger generated neutral recombination clusters yielded $\Omega_{(0-1) /(1-2)}=1.60$ which corresponds to a mobility diame-
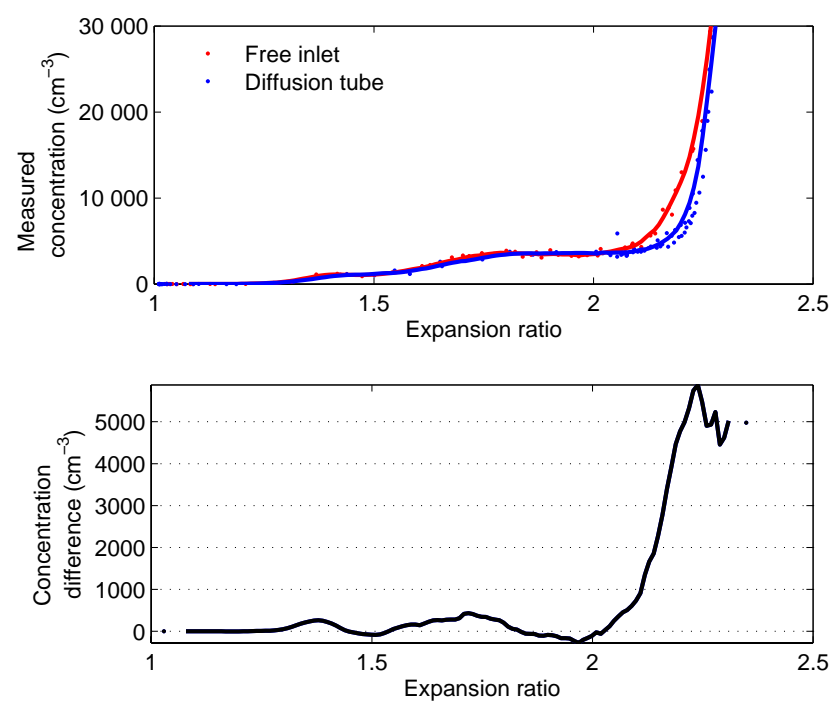

Fig. 9. Data displayed as in Fig. 10 for an expansion scan experiment on 28 May 2007 at 11:02-11:33. Water is used as a condensing vapour.

ter of $1.9 \mathrm{~nm}$. From the standard deviation of $\Omega_{(0-1) /(1-2)}$ we get a lower end error estimate for the diameter to be ca. $1.6 \mathrm{~nm}$. Using the standard deviation of $\Omega_{(0-1) /(1-2)}$ no realistic estimation for an upper limit of the particle size can be obtained, but it is clear from the pulse height spectra and the supporting data that the clusters observed during non-event times have to be smaller than $3 \mathrm{~nm}$ in diameter. Besides the relatively large scattering of $\Omega_{(0-1) /(1-2)}$, also the error arising from the diffusion battery calibration inaccuracies is obviously quite high with such small particle sizes. Furthermore, it should be emphasized that the determined diameter of ca. $1.8 \mathrm{~nm}$ is an average size of the detected clusters, and is not necessarily equal to the true average size of the whole cluster pool. The counting efficiency of the PH-CPC can be strongly enhanced for the largest clusters. Therefore the $1.8 \mathrm{~nm}$ given here should be taken only as suggestive.

Two examples from the E-CPC measurements are presented in Figs. 8 and 9. Expansion scans with water and butanol as a working fluid both yielded qualitatively the same results. Unfortunately with the current setup simultaneous measurement with butanol and water was not possible and therefore no indication of e.g. solubility or composition of these clusters can be obtained from the data.

Large particles activate first after which there is a plateau in the concentration curve (Figs. 8 and 9). Finally, the concentration starts to increase rapidly. The difference between the experiments where the cluster-sized objects were either present or removed by the diffusion tubes (see Sect. 2.3) is clear. The concentration difference between these two curves around the onset of homogeneous nucleation gives directly the concentration of clusters activated inside the expansion chamber. The signal is rapidly masked by fluctuations when 


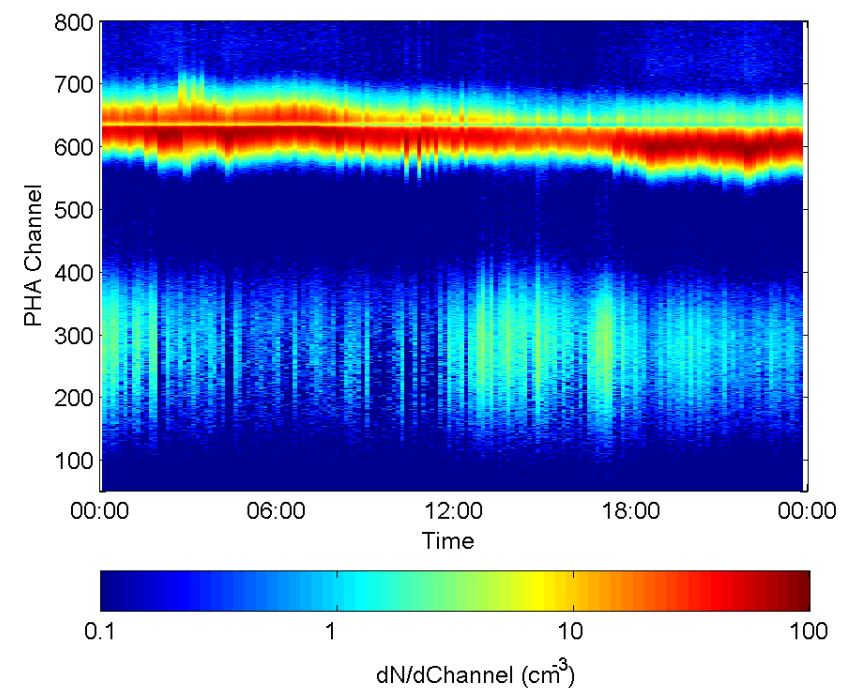

Fig. 10. Pulse height spectra recorded during a non-event day (12 June 2007). Large particles are accumulating in the channels around 600. Clusters are seen in around channel 300. Detection efficiency or losses are not taken into account. Gap around the channel 630 is an instrumental artefact.

the concentration starts to increase further. The nucleation rate is highly sensitive to several parameters, such as temperature and saturation ratio which are not controlled in high enough accuracy. That causes large fluctuations after homogeneous nucleation starts. It is noteworthy that the activation of clusters takes place at the same expansion ratios as the activation of the laboratory generated recombination clusters (Fig. 4). This supports the assumption that the observed signal really is due to the cluster activation and not e.g. multicomponent homogeneous nucleation.

Few experiments with the ion filter at the inlet were conducted but the high fluctuations covered the possible signal from the ion activation. This was expected, since close to the onset of homogeneous nucleation the fluctuations, or noise, in the signal were typically $\sim 1000 \mathrm{~cm}^{-3}$. Assuming e.g. 5$10 \%$ detection efficiency for the ions would lead to signals by a factor of 5-10 smaller than the noise level with the typical cluster ion concentrations $\left(\sim 2000 \mathrm{~cm}^{-3}\right)$. Thus, extracting the ion signal from this limited data set was not possible.

\section{June 2007}

Before any indication of new particle formation was observed by the DMPS, the PH-CPC detected clusters at sizes below the detection limit of the DMPS (Fig. 6). Another example of the detected cluster pool is shown in Fig. 10. Data is recorded during a non-event day on 12 June 2007. The figure shows a difference in signals measured with diffusion battery stages 0 and 3 . The detection efficiency or losses in the zeroth stage are not accounted for. Throughout the day a clear signal around the MCA channel 300 was observed sug-

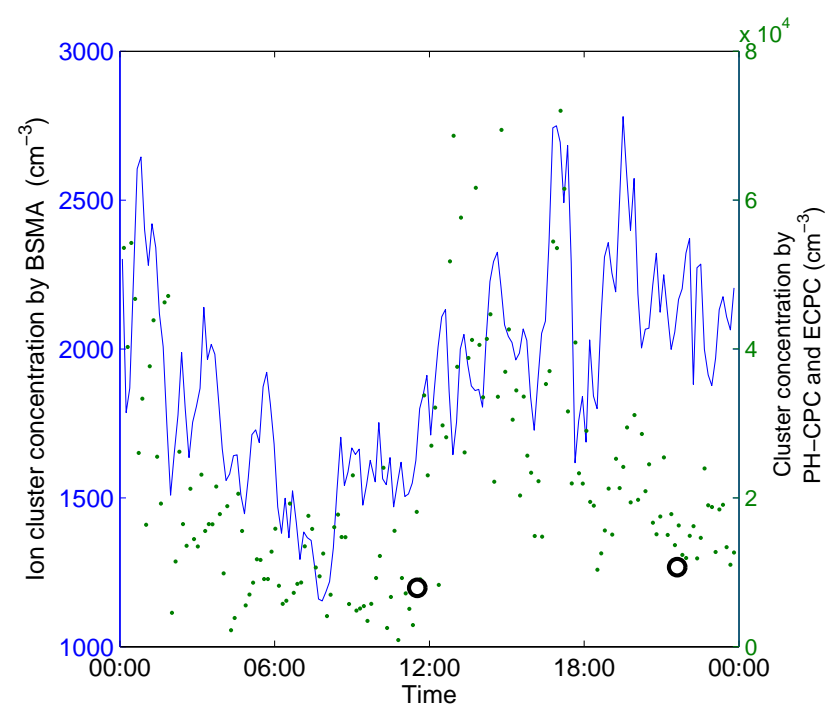

Fig. 11. Total cluster concentrations measured with the PH-CPC (green dots) and by the E-CPC using butanol as a working fluid (black circles), and ion cluster concentration measured with the BSMA (blue line) on 12 June 2007. Detection efficiency of $2 \%$ is assumed for the PH-CPC and 13\% for the E-CPC. The PH-CPC and the BSMA signals seem to correlate with each other. Variation in the PH-CPC signal, however, is much larger than the scattering in the BSMA data.

gesting a continuous presence of clusters. Larger particles (not affected by the diffusion battery) are once again accumulating in the channels around 600.

Figure 11 shows total cluster concentrations measured by the PH-CPC and the E-CPC, and the ion cluster concentration measured by the BSMA on 12 June 2007 (same day as in Fig. 10). For the PH-CPC the detection efficiency of $2 \%$ and the diffusion battery 0 -stage penetration of $40 \%$ (1.8 nm particles) are assumed. The detection efficiency of the E-CPC for the artificial charger generated clusters was 6-7 times higher than the corresponding efficiency of the PH-CPC. The detection efficiency was within the measurement accuracy independent of the working fluid indicating that the detection efficiency of the E-CPC with the scanning technique is mainly determined by nanoparticle transport losses from the inlet to the expansion chamber rather than the activation probability. Therefore the detection efficiency of $13 \%$ was assumed for the E-CPC with both working fluids. It should be emphasized that these are only rough estimates and the true detection efficiencies can be significantly different. However, it is clearly seen in Fig. 11 that the order of magnitude is the same in both the PH-CPC and in the E-CPC measurements. Similarities in diurnal behaviour between the total cluster concentration detected by the PH-CPC and the ion cluster concentration can be seen. However, a variation in the total cluster concentration is much higher than in the ion cluster concentration. 


\section{Discussion}

Both the pulse height and the expansion CPC measurements showed that besides large ( $>3 \mathrm{~nm}$ ) particle activation and homogeneous self-nucleation of the working fluid, there is a third process taking place in the supersaturated conditions inside the PH-CPC condenser and the E-CPC expansion chamber. This process was concluded to be most likely neutral cluster activation (see also Kulmala et al., 2006; Kulmala et al., 2007a, 2007b). Several factors provide evidence in favour of this process. First, ion clusters alone cannot explain the observed signal as shown for example in Fig. 7. Second, the size of the clusters determined from the diffusion battery measurements was reasonably large, around $1.8 \mathrm{~nm}$ in mobility equivalent diameter. Such a size would correspond to an object with a mass of approximately $1000 \mathrm{amu}$ (Kilpatrick, 1971; Mäkelä et al., 1996). Such a mass could, in principle, be exceeded by large organic molecules. It means that with the current measurement setup we are approaching molecular sizes in the nanoparticle detection.

The data does not directly show that the observed (neutral) clusters were thermodynamically stable (Kulmala, et al., 2000; Vehkamäki, 2004). However, since the composition of surrounding gas changes when the clusters enter the PH-CPC condenser $(90 \%$ of the sample air is filtered and saturated with butanol vapour before it is directed to the condenser), it is reasonable to assume that they were at least to some extent stable or slowly growing dynamic clusters. Survival probability of unstable clusters from the inlet to the point of activation is expected to be rather poor. Also, since similar (within a factor $\sim 5$ ) concentrations were detected with two instruments with a different design and operation principle, it would be very surprising if the survival probabilities of unstable weakly bonded clusters were equal in both of the instruments.

If the observed signal is due to cluster activation inside the $\mathrm{CPC}$, can we then conclude something about the formation mechanism of these clusters? Variation of the total cluster concentration measured with the PH-CPC is clearly stronger (factor of $\sim 10$ ) than the variation of the ion concentration measured by the BSMA (factor of $\sim 2$ ) but the similarities in the diurnal cycles can be seen. This is partly due to the fact that the neutral and the ion cluster concentrations should both be dependent on the coagulation sink provided by the larger aerosol particles. From Fig. 11 it seems that the cluster concentration is higher during night time indicating that the source of neutral clusters is stronger on the surface layer since, after the boundary layer mixing in the morning the concentration goes down. This observation suggests that the cluster formation rate is dependent either on the atmospheric ion concentration, which is higher during night-time because of an elevated radon level, or biological activities. However, because of numerous uncertainties influencing the PH-CPC detection efficiency, this observation is still very uncertain.

Ion-ion recombination is also expected to form stable clus- ters. Kulmala et al. (2007a), however, showed that the concentration of the clusters formed via recombination is typically from few tens up to few hundreds of clusters $\mathrm{cm}^{-3}$, while our estimation for typical neutral cluster concentrations ranged from some thousands up to few tens of thousands of clusters $\mathrm{cm}^{-3}$. Thus also other cluster formation pathways possibly involving for example organic oxidation products (Kurtén et al., 2007) are needed. A detailed analysis and comparisons to precursor concentrations, meteorological parameters, etc. are needed before we can say more on the origin of these clusters.

\section{Conclusions}

We have investigated the performance of two different CPC systems in atmospheric cluster detection, and in practise we were able to detect atmospheric sub-2 nm clusters using the PH-CPC and the E-CPC. Since the existence of these clusters has been observed very recently (Kulmala et al., 2007a), more measurements with the different instruments were required to ensure their existence and also to quantify their size and concentration. Using two parallel instruments we were able to verify the existence of neutral clusters and also we were able to observe them continuously. Furthermore, the observed size and concentrations gave similar (but not identical) values using the two instruments. The observed cluster size was around $1.5-2 \mathrm{~nm}$ in mobility equivalent diameter. The concentration of clusters was typically ca. 5-10 times higher than the ion cluster concentration and also 2-5 times higher than the concentration of the sum of ion clusters and recombination clusters. Therefore a pool of neutral clusters seems to exist in the boreal forest environment and these results support our recent results (Kulmala et al., 2007a).

The measured cluster concentrations are still very uncertain and more detailed investigations are needed to improve the instruments, and to accurately calibrate and test them. In any case we can consider the values given here as lower limits of the cluster concentration, since we were most probably not able to observe neutral clusters with sizes approaching $1 \mathrm{~nm}$.

The origin of the observed clusters is still open. They can be thermodynamically stable clusters like ammonium bisulphate clusters proposed by Vehkamäki et al. (2004) or they can be slowly growing dynamic clusters. Some of them could even be "snapshot clusters" forming and decaying dynamically and existing only for a short period during the observation. However, those clusters are supposed to be activated (e.g. Kulmala et al., 2006) probably with sulphuric acid. A main mechanism for atmospheric nucleation would then be the activation of the more or less permanently existing clusters. This mechanism is straightforward to apply in global models (e.g. Spracklen et al., 2006). 
Acknowledgements. This work has been partially funded by European Commission 6th Framework programme project EUCAARI, contract no 036833-2 (EUCAARI). Also Maj and Tor Nessling foundation is acknowledged for financial support.

Edited by: A. Wiedensohler

\section{References}

Aalto, P., Hämeri, K., Becker, E., Weber, R., Salm, J., Mäkelä, J. M., Hoell, C., O’Dowd, C. D., Karlsson, H., Hansson, H.-C., Väkevä, M., Koponen, I. K., Buzorius, G., and Kulmala, M.: Physical characterization of aerosol particles in boreal forests. Tellus 53B, 344-358, 2001

Baron, P. A. and Willeke, K.: Aerosol Measurement - Principles, Techniques, and Applications (2nd edition), John Wiley and Sons, New York, pp. 1131, 2001.

Curtius, J.: Nucleation of Atmospheric Aerosol Particles, Comptes Rendus Physique, 7, 1027-1045, doi:10.1016/j.crhy.2006.10.018, 2006

Dick, W. D., McMurry, P. H., Weber, R. J., and Quant, R.: Whitelight detection for nanoparticle sizing with the TSI Ultrafine Condensation Particle Counter, J. Nanoparticle Res., 2, 85-90, 2000.

Hanson, D. R., Eisele, F. L., Ball, S. M., and McMurry, P. M.: Sizing small sulfuric acid particles with an ultrafine particle condensation nucleus counter, Aerosol Sci. Technol., 36, 554-559, 2002.

Hari, P. and Kulmala, M.: Station for measuring ecosystematmosphere relations (SMEAR II), Boreal Environ. Res., 10, 315-322, 2005.

Hirsikko, A., Laakso, L., Hõrrak, U., Aalto, P. P., Kerminen, V.M., and Kulmala, M.: Annual and size dependent variation of growth rates and ion concentrations in boreal forest, Boreal Environ. Res, 10, 357-369, 2005.

Kerminen, V.-M., Pirjola, L., and Kulmala M.: How significantly does coagulational scavenging limit atmospheric particle production?, J. Geophys. Res., 106(24), 119-126, 2001.

Kilpatrick, W. D.: An experimental mass-mobility relation for ions in air at atmospheric pressure, Proc. Annu. Conf. Mass Spectrosc., 19, 320-325, 1971.

Kulmala, M., Pirjola, L., and Mäkelä, J. M.: Stable sulphate clusters as a source of new atmospheric particles, Nature, 404, 66-69, 2000.

Kulmala, M.: How particles nucleate and grow?, Science, 302, 1000-1001, 2003.

Kulmala, M., Vehkamäki, H., Petäjä, T., Dal Maso, M., Lauri, A., Kerminen, V.-M., Birmili, W., and McMurry, P. H.: Formation and growth rates of ultrafine atmospheric particles: a review of observations, J. Aerosol Sci., 35, 143-176, 2004.

Kulmala M., Lehtinen K. E. J., Laakso, L., Mordas, G., and Hämeri, K.: On the existence of neutral atmospheric clusters, Boreal Environ. Res., 10, 79-87, 2005.

Kulmala, M., Lehtinen, K. E. J., and Laaksonen, A.: Cluster activation theory as an explanation of the linear dependence between formation rate of $3 \mathrm{~nm}$ particles and sulphuric acid concentration, Atmos. Chem. Phys., 6, 787-793, 2006, http://www.atmos-chem-phys.net/6/787/2006/.
Kulmala, M. and Tammet, H.: Finnish-Estonian air ion and aerosol workshops, Boreal Environ. Res. 12, 237-245, 2007.

Kulmala, M., Riipinen, I., Sipilä, M., Manninen, H., Petäjä, T., Junninen H., Dal Maso, M., Mordas, G., Mirme, A., Vana, M., Hirsikko, A., Laakso, L., Harrison, R. M., Hanson, I., Leung, C., Lehtinen, K. E. J., and Kerminen, V.-M.: Towards direct measurement of atmospheric nucleation, Science, 318, 89-92, doi:10.1126/science.1144124, 2007a.

Kulmala, M., Mordas, G., Petäjä, T., Grönholm, T., Aalto, P. P., Vehkamäki, H., Hienola, A. I., Herrmann, E., Sipilä, M., Riipinen, I., Manninen, H. E., Hämeri, K., Stratmann, F., Bilde, M., Winkler, P. M., Birmili, W., and Wagner, P. E.: The condensation particle counter battery (CPCB): A new tool to investigate the activation properties of nanoparticles, J. Aerosol Sci., 38, 289-304, 2007b.

Kurtén, T., Bonn, B., Vehkamäki, H., and Kulmala, M.: Computational study of the reaction between biogenic stabilized Criegee intermediates and sulfuric acid, J. Phys. Chem. A., 111, 3394 3401, 2007.

Kürten, A., Curtius, J., Nillius, B., and Borrmann, S.: Characterization of an automated, water-based expansion condensation nucleus counter for ultrafine particles, Aerosol Sci. Technol., 39, 1174-1183, 2005.

Lyubovtseva, Y. S., Sogacheva, L., Dal Maso, M., Bonn, B., Keronen, P., and Kulmala, M.: Seasonal variations of trace gases, meteorological parameters, and formation of aerosols in boreal forests, Boreal Environ. Res., 10, 493-510, 2005.

Marti, J. J., Weber, R. J., Saros, M. T., Vasilou, J. G., and McMurry, P. H.: Modification of the TSI 3025 Condensation Particle Counter for pulse height analysis, Aerosol Sci. Technol., 25, 214-218, 1996.

Mertes, S. Schröder, F., and Wiedensohler, A.: The particle detection efficiency curve of the TSI-3010 CPC as a function of temperature difference between saturator and condenser, Aerosol Sci. Technol., 23, 257-261, 1995.

Mäkelä, J. M., Jokinen, V., Mattila, T., Ukkonen, A., and Keskinen, J.: Mobility distribution of acetone cluster ions, J. Aerosol Sci., 27, 175-190,1996.

O’Dowd, C. D., Aalto, P., Hämeri, K., Kulmala, M., and Hoffmann, T.: Atmospheric particles from organic vapours, Nature, 416, 497-498, 2002.

Saros, M., Weber, R. J., Marti, J., and McMurry, P. H.: Ultra fine aerosol measurement using a condensation nucleus counter with pulse height analysis, Aerosol Sci. Technol., 25, 200-213, 1996.

Spracklen, D. V., Carslaw, K. S., Kulmala, M., Kerminen, V.-M., Mann, G. W., Sihto, S.-L.: The contribution of boundary layer nucleation events to total particle concentrations on regional and global scales, Atmos. Chem. Phys. 6, 5631-5648, 2006.

Stoltzenburg, M. R. and McMurry, P. H.: An ultrafine aerosol condensation nucleus counter, Aerosol Sci. Technol. 14, 48-65, 1991.

Tammet, H.: Balanced scanning mobility analyzer BSMA, in: Nucleation and atmospheric aerosols 2004, edited by: Kasahara, M., and Kulmala, M., 16th International Conference, Kyoto University Press, Japan, 294-297, 2004.

Tammet, H. and Kulmala, M.: Simulation tool for atmospheric aerosol nucleation bursts, J. Aerosol Sci., 36, 173-196, 2005.

Tammet, H.: Continuous scanning of the mobility and size distribution of charged clusters and nanometer particles in atmospheric 
air and the balanced scanning mobility analyzer BSMA, Atmos. Res., 82, 523-535, 2006.

Vehkamäki, H., Napari, I., Kulmala, M., and Noppel, M.: Stable ammonium bisulphate clusters in the atmosphere. Phys. Rev. Lett. 93, 148501, doi:10.1103/PhysRevLett.93.14850, 2004.

Wagner, P. E.: A constant-angle Mie scattering method (CAMS) for investigation of particle formation processes, J. Colloid Interface. Sci., 105, 456-467, 1985.

Weber, R. J., McMurry, P. H., Eisele, F. L., and Tanner, D. J.: Measurement of expected nucleation precursor species and 3-500-nm diameter particles at Mauna Loa observatory, Hawaii, J. Atmos. Sci., 52, 2242-2257, 1995.
Weber, R. J., Stolzenburg, M. R., Pandis, S. N., and McMurry, P. H.: Inversion of ultrafine condensation nucleus counter pulse height distributions to obtain nanoparticle $(\sim 3-10 \mathrm{~nm})$ size distributions, J. Aerosol Sci., 29, 601-615, 1998.

Winkler, P. M., Steiner, G., Vrtala, A., Vehkamäki, H., Noppel, M., Lehtinen, K. E. J., Reischl, G. P., Wagner, P. E., and Kulmala, M.: Heterogeneous nucleation experiments bridgin scale from molecular ion clusters to nanoparticles, Science, 319, 13741377, 2008. 